\title{
Laparoscopic esophagogastric circular stapled anastomosis: a modified technique to protect the esophagus
}

\author{
Naoki Hiki, Tetsu Fukunaga, Toshiharu Yamaguchi, Souya Nunobe, Masanori Tokunaga, \\ Shigekazu Ohyama, Yasuyuki Seto, and Tetsuichiro Muto \\ Department of Gastroenterological Surgery, Gastroenterological Center, Cancer Institute Hospital, Japanese Foundation for Cancer \\ Research, 3-10-6 Ariake, Koto-ku, Tokyo 135-8550, Japan
}

\begin{abstract}
Laparoscopic surgery is increasingly being applied to gastric cancer surgery, including proximal gastrectomy for the resection of cancer located in the upper gastric body. Despite the ease of use of stapling devices for end-to-end anastomosis, esophagogastric anastomosis is complicated by the narrow laparoscopic space, making the placement of an esophageal purse-string suture and anvil insertion into the fragile and contracted esophagus difficult. The aim of this study was to employ a novel esophagogastric anastomosis technique for laparoscopic surgery which may avoid esophageal breakdown. Eleven patients with early gastric cancer within the upper gastric body underwent laparoscopic proximal gastrectomy. The anvil of the stapler was introduced into the esophagus through a small gastrostomy, before transection of the esophagus. The esophageal-to-anterior gastric wall anastomosis was performed using a double-stapling technique, without the need to apply a purse-string suture. The mean operation time was $237 \pm 15 \mathrm{~min}$ and estimated blood loss was $39 \pm 21 \mathrm{ml}$. The postoperative course was uneventful in all 11 patients, with no anastomotic leakage observed. Two patients needed endoscopic balloon dilation of an anastomotic stricture 24 to 28 days postoperatively. This modified procedure of laparoscopic esophagogastric anastomosis after proximal gastrectomy for the resection of cancer is a simple, rapid, and atraumatic technique which reduces the risk of anastomotic insufficiency.
\end{abstract}

Key words Surgical staplers · Anastomosis · Laparoscopy

\section{Introduction}

Esophageal anastomosis has been facilitated by the development of the inverting end-to-end anastomosis (EEA) mechanical stapling device $[1,2]$. Although the

Offprint requests to: $\mathrm{N}$. Hiki

Received: May 21, 2007 / Accepted: July 13, 2007
EEA stapler is known as a technically excellent tool with very low failure or leakage rates, the anastomotic leakage of an esophageal anastomosis may lead to a fatal outcome. An often troubling technical problem is the difficulty of inserting the anvil from the EEA stapler into the transected esophageal lumen after placing a purse-string suture. Excessive force employed to insert the anvil into a noncompliant esophagus may result in mucosal tears and retraction, causing a technical failure and disastrous anastomotic leakage that may not be recognized until days later [3]. In laparoscopic surgery, both the placement of the purse-string suture and anvil insertion are even more difficult than in open surgery, because of the narrow working space. However, a widely accepted standard technique for esophageal anastomosis during laparoscopic surgery has yet to be established. Although other techniques, such as the transoral passage of the anvil as well as using EEA have been reported, the size of the anvil head was small, and this may cause postoperative stricture [4].

The present study describes a novel, double-stapling anastomotic technique especially useful for laparoscopic gastric surgery that eliminates the need to apply a pursestring suture and reduces the difficulty of large-size anvil insertion, thus avoiding subsequent esophageal breakdown. The technique was employed in 11 patients, as described below.

\section{Patients and methods}

Operative technique for the esophagogastric anastomosis

After completion of lymph node dissection of the greater and lesser curvatures of the stomach, and the stomach was ready for proximal gastrectomy, the anterior wall of the upper gastric area was incised using ultrasonically activated coagulation devices (AutoSonix 
and LigaSure; Tyco Healthcare Japan, Tokyo, Japan). The nasogastric tube was recognized and easily pulled out through the incision on the stomach wall into the abdominal cavity.

A 3- to 4-cm vertical incision was then made in the left abdominal wall. A wound retractor was placed into the wound as an operation port and the nasogastric tube was exteriorized through this site.

The head of the anvil was prepared with 2-0 Ti-Cron sutures (Coviden, Norwalk, CT, USA) and the tail of the anvil was capped with a $10-\mathrm{cm}$ length of nasogastric tube (Fig. 1A). The anvil sutures were then tied to the nasogastric tube (Fig. 1B). The incision in the anterior gastric wall was extended towards the esophagogastric junction (EGJ), but not past the EGJ. To prevent splitting of the esophageal tissue during anvil insertion, the EGJ should be kept intact during this part of the procedure. The nasogastric tube was then gently pulled by the anesthetist while the tail of the anvil was pushed into the gastric lumen by the grasper of the operator. This procedure makes insertion of the anvil smooth and atraumatic. When the tail of the anvil reached the esophagus, the incision was further extended with the LigaSure (Tyco Healthcare Japan) and the EGJ was separated. The length of nasogastric tube connected to the tail of the anvil was lifted up (Fig. 2A) and the distal esophagus was transected using an Endo GIA Universal 60-mm stapler (Tyco Healthcare Japan; Fig. 2B). The anvil was then positioned correctly by pulling on the length of tubing. To keep the entry hole of the anvil as tight as possible, the esophageal incision was tightly clamped shut by using both assistants' graspers. To avoid the risk of "gap" formation on the posterior side of the staple line, the $10-\mathrm{cm}$-length of nasogastric tube connected with the tail of the anvil was lifted up cranially as far as possible during the transection of the distal esophagus. This approach makes it possible for the anvil head to be replaced correctly into the esophagus; therefore, an additional purse-string suture is not necessary for this procedure.

The stomach was exteriorized through the left abdominal incision, and a partial gastrectomy was performed. The greater curvature of the stomach was opened for insertion of the main body of the mechanical circular stapler [Premium Plus CEEA (PCEEA); Coviden], and, after a purse-string suture was placed, the anvil was inserted into the remnant stomach, and the anterior wall of the lesser curvature of the stomach was punctured by the tip of the PCEEA (Fig. 3A). The remnant stomach was then returned to the abdominal cavity while the shaft of the PCEEA remained externally where it could be controlled from the outside of the abdomen. A pneumoperitoneum was created for anastomosis. The esophagogastric anastomosis was performed using a 25- or 28-mm-diameter PCEEA (Fig.

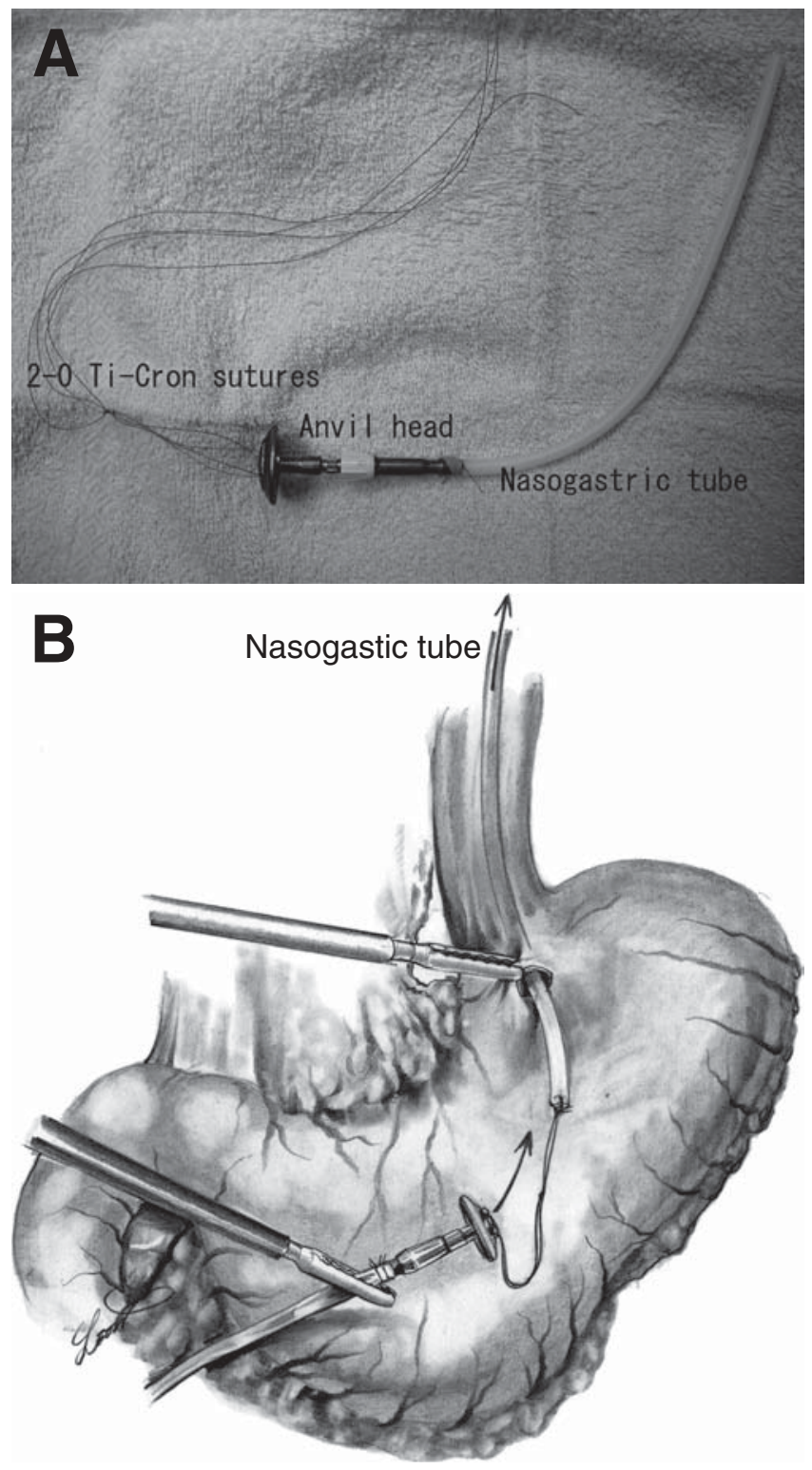

Fig. 1A,B. A Anvil head preparation. The head of the anvil was prepared with 2-0 Ti-Cron sutures and the tail of the anvil was capped with a $10-\mathrm{cm}$ length of nasogastric tube. B The anvil sutures were then tied to the nasogastric tube

3B). The gastrostomy was closed using an Endo GIA Universal 60-mm stapler (Tyco Healthcare Japan; Fig. $3 \mathrm{C})$.

\section{Patients}

From May to December 2005, an esophagogastric anastomosis with laparoscopic proximal gastrectomy, described above, was performed in 11 patients with early gastric cancer by surgeons in the Department of Gastrointestinal Surgery at the Cancer Institute, Tokyo, Japan. Histologically, the tumors in these 11 patients 


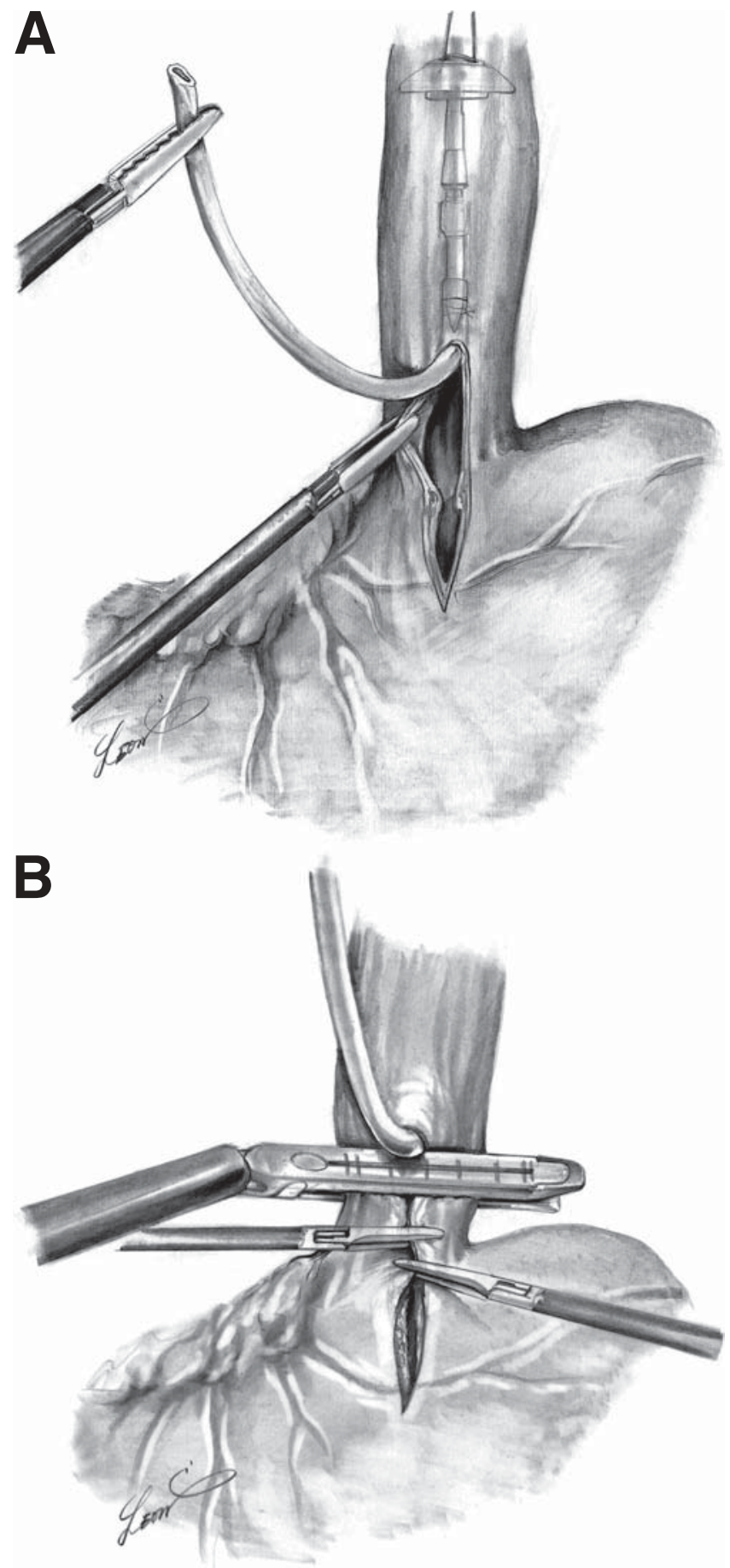

Fig. 2A,B. Esophageal transection. A When the tail of the anvil reached the esophagus, the incision was further extended with a LigaSure device (Tyco Healthcare Japan) and the esophagogastric junction (EGJ) was separated. The length of nasogastric tube connected to the tail of the anvil was lifted up. B The distal esophagus was transected using an Endo GIA Universal 60-mm stapler (Tyco Healthcare Japan) were classified as adenocarcinomas that had invaded only the mucosa or submucosa of the stomach without lymph node metastasis. The clinical classification of tumor depth (cT) and nodal involvement $(\mathrm{cN})$ was determined by preoperative and intraoperative evaluation, including barium radiography, upper gastrointestinal tract endoscopy, abdominal ultrasonography, computed tomography, and endoscopic ultrasonography. The intraoperative $\mathrm{cN}$ classification was evaluated by node palpation. The surgical method we have described is indicated for intramucosal or submucosal carcinoma without lymph node metastasis (cT1, cN0). Patients with early gastric cancer not suitable for endoscopic mucosal resection (EMR), such as patients with submucosal cancer and mucosal cancer that was histologically confirmed as poorly differentiated adenocarcinoma were treated with this procedure. In all 11 patients, the tumors were located in the cardia or upper third of the stomach and these tumors were indicated for proximal partial gastrectomy (about $30 \%$ of the stomach).

Details of the following surgical parameters were collected: operation time, estimated blood loss, and degree of lymph node dissection. Postoperative data recorded included gastric fullness (when conditions such as upper abdominal distension, remnant stomach fullness on Xray, and obstruction for longer than $24 \mathrm{~h}$ were fulfilled), anastomotic complications (leakage, stenosis, bleeding, and ulceration), ileus, time until start of oral intake, and length of postoperative hospital stay.

\section{Results}

Summaries of the patients' clinical histories are shown in Table 1. Age, sex, body mass index, and clinical

Table 1. Characteristics of patients undergoing proximal gastrectomy with new anastomotic technique

\begin{tabular}{lc}
\hline & $n=11$ \\
\hline $\begin{array}{l}\text { Number of patients } \\
\text { Sex }\end{array} \quad 11$ \\
$\quad$ Male/Female & $5 / 6$ \\
Age (years) & \\
$\quad$ Mean & $66 \pm 4$ \\
$\quad$ Range & $37-84$ \\
Body mass index $\left(\mathrm{kg} / \mathrm{m}^{2}\right)$ & $22 \pm 1$ \\
Preoperative complication & \\
$\quad$ Diabetes & $1(9 \%)$ \\
Ischemic heart disease & $0(0 \%)$ \\
Liver cirrhosis & $0(0 \%)$ \\
Hypertension & $1(9 \%)$ \\
Clinical stage & $10(91 \%)$ \\
IA & $1(9 \%)$ \\
IB &
\end{tabular}

Data values are presented as means \pm SE. Body mass index $=$ body weight $/$ height $^{2}\left(\mathrm{~kg} / \mathrm{m}^{2}\right)$ 


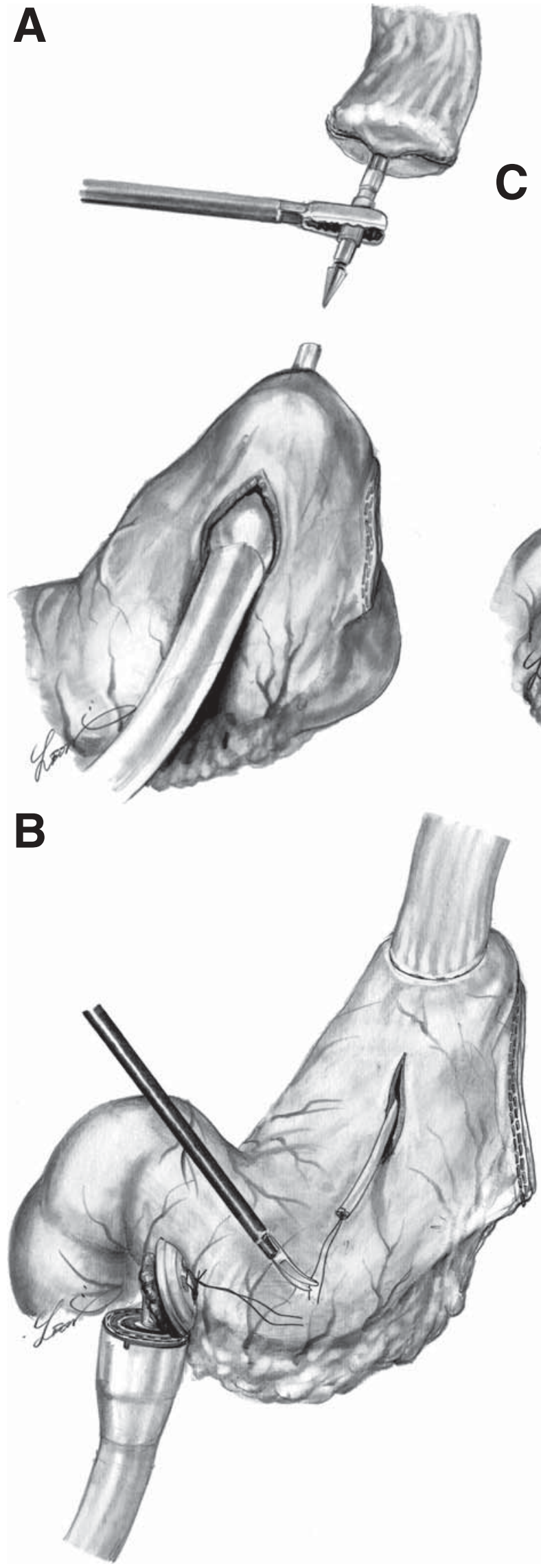

Fig. 3A-C. Esophago-gastric anastomosis. A The stomach was exteriorized through the left abdominal incision, and a partial gastrectomy was performed. The greater curvature of the stomach was opened for insertion of the main body of the mechanical circular stapler (PCEEA), the anvil was inserted into the remnant stomach, and the anterior wall of the lesser curvature of the stomach was punctured by the tip of the PCEEA. B The remnant stomach was then returned to the abdominal cavity while the shaft of the PCEEA remained externally where it could be controlled from the outside of the abdomen. A pneumoperitoneum was created for anastomosis. The esophagogastric anastomosis was performed using a 25or 28-mm diameter PCEEA. C The gastrostomy was closed using an Endo GIA Universal 60-mm stapler (Tyco Healthcare Japan) 
Table 2. Operative data from patients undergoing proximal gastrectomy with new anastomotic technique

\begin{tabular}{lc}
\hline & $n=11$ \\
\hline Operation time (min) & $237 \pm 15$ \\
Intraoperative blood loss (ml) & $39 \pm 21$ \\
Total number of resected lymph nodes & $22 \pm 3$ \\
Conversion to open surgery & $0(0 \%)$ \\
Postoperative complications & \\
$\quad$ Gastric fullness & $0(0 \%)$ \\
Anastomotic leakage & $0(0 \%)$ \\
Anastomotic stenosis & $2(18 \%)$ \\
Anastomotic bleeding & $0(0 \%)$ \\
Anastomotic ulceration & $0(0 \%)$ \\
$\quad$ Ileus & $0(0 \%)$ \\
Time until start of oral intake (days) & $2.1 \pm 0.1$ \\
Postoperative hospital stay (days) & $15.1 \pm 1.3$ \\
\hline
\end{tabular}

Data values are presented as means $\pm \mathrm{SE}$

staging of all patients are reported, with $91 \%$ of patients diagnosed as stage IA. Table 2 shows a summary of the operative data. The mean operation time for the procedure was $237 \pm 15 \mathrm{~min}$, mean estimated blood loss was $39 \pm 21 \mathrm{ml}$, and the mean total number of dissected lymph nodes was $22 \pm 3$. None of the patients required conversion to open surgery. The postoperative course was uneventful in all patients and no anastomotic leakage was observed in any of the patients. Two patients needed endoscopic balloon dilation of an anastomotic stricture, 24 to 28 days postoperatively. The mean time until start of oral intake was $2.1 \pm 0.1$ days and the mean length of postoperative hospital stay was $15.1 \pm 1.3$ days.

\section{Discussion}

Stapling devices for EEA have facilitated more rapid and reliable reestablishment of esophagogastric continuity following esophageal resections [1]. Although the EEA stapler allows technically excellent anastomoses to be reproducibly performed with very low failure or leakage rates [5], various intraoperative problems can arise, especially with the proper placement of the esophageal purse-string suture or insertion of the anvil into the fragile, commonly contracted lumen [3].

An often troubling technical problem is the difficulty of inserting the anvil from the EEA stapler into the transected esophageal lumen, as this procedure may cause mucosal tears and anastomotic leaks [3]. Many attempts have been made to dilate the lumen against the contraction of the esophageal muscle. The muscular contraction which is caused by the manipulation of esophageal tissues can be ameliorated by intravenous glucagon injection. Exogenous glucagon decreases resting lower esophageal sphincter pressure [6] and acts as a hypotonic spasmolytic agent for overcoming smooth muscle spasm in most of the alimentary tract [7]. A Foley catheter with a 30-ml balloon can be blown up gently inside the proximal esophagus, after the use of glucagon, to dilate the lumen. A clamp can also be used to dilate the lumen, but the resultant uneven distension of the lumen tends to tear the mucosa [3]. Nevertheless, the best solution to prevent muscle contraction of the esophagus is to abolish any manipulation of the esophagus. The new technique we have described makes insertion of the anvil possible without any manipulation of the esophagus. Before transection of the esophagus, only the anterior wall of the stomach is opened for the entry hole of the anvil, and after the introduction of the anvil the esophagus is transected. The excessive force normally used to insert the anvil into the dissected esophagus may cause tearing of the esophageal muscle fiber and mucosa, a critical factor in anastomotic complications. Thus, another advantage of our procedure is the maintenance of the EGJ until after anvil insertion, as the remnant EGJ effectively prevents the esophageal muscle and mucosal tears. Moreover, the insertion force on the anvil is reduced by both pushing the tail and pulling the head of the anvil via the nasogastric tube, allowing insertion of the anvil beyond the remnant EGJ. A new commercially available device with the anvil introduced through the mouth into a nasogastric tube permits the performance of an esophagogastric stapled anastomosis. Although this method theoretically permits an esophagogastric stapled anastomosis with a $25-\mathrm{mm}$ EEA, sometimes difficulties are faced in introducing the anvil to the lower esophagus, because of some narrow spaces, such as the larynx and esophagus, at the tracheal bifurcation level. Thus, in this method, esophageal mucosal injury could be caused by a larger-sized EEA. Our new technique has made it possible to introduce a larger-sized anvil without any risk of esophageal mucosal injury.

The stricture rate with a stapler anastomosis was reported to be high [8]. Although there has been no significant evidence which showed a correlation between the diameter of the stapler and anastomotic diameter, the stapler size may be correlated to the anastomotic diameter and increase in body weight after gastrectomy [9]. Indeed, our two patients with anastomotic stricture had a small anastomotic diameter $(25 \mathrm{~mm})$, but even these two patients required only two to three dilations with an endoscopic pneumatic balloon. It has been reported that other techniques such as the transoral passage of the anvil, as well as using EEA, do not cause injury to the esophagus; however, in that study, the size of the anastomotic diameter was limited, at up to $25 \mathrm{~mm}$ [4]. The use of this technique frequently permits a larger stapler (usually $28-\mathrm{mm}$ ) to be inserted. 
Because of anatomical limitations at the EGJ after proximal gastrectomy, gastroesophageal reconstruction is technically challenging. The placement of a pursestring suture and the insertion of an anvil are problematic, due to the difficult exposure at the base of the mediastinum within a narrow space which can only be created by pneumoperitoneum. The esophagogastric anastomotic procedure following laparoscopic gastrectomy described in this study eliminates the need to apply a purse-string suture in the esophagus, uses a double-stapling technique and gentle anvil insertion, and is safe. Although this technique is described for esophagogastric anastomosis, the technique may also apply to other types of surgery, such as an esophagojejuno anastomosis in total gastrectomy.

Acknowledgments All figures were drawn by special medical illustrator Mr. Leon Sakuma. We are deeply indebted to Miss Noriko Okita for checking the Englishlanguage usage in the text.

\section{References}

1. Steichen FM, Ravitch MM. Mechanical sutures in esophageal surgery. Ann Surg 1980;191:373-81.
2. Ravitch MM, Steichen FM. A stapling instrument for end-to-end inverting anastomoses in the gastrointestinal tract. Ann Surg 1979;189:791-7.

3. Robinson LA, Moulton AL, Fleming WH. Techniques to simplify esophagogastric circular stapled anastomoses. J Surg Oncol 1994;57:266-9.

4. Waage A, Gagner M, Feng JJ. Early experience with computermediated flexible circular stapling technique for upper gastrointestinal anastomosis. Obes Surg 2003;13:88-94.

5. Peracchia A, Bardini R, Ruol A, Asolati M, Scibetta D. Esophagovisceral anastomotic leak. A prospective statistical study of predisposing factors. J Thorac Cardiovasc Surg 1988;95:685-91.

6. Hogan WJ, Dodds WJ, Hoke SE, Reid DP, Kalkhoff RK, Arndorfer RC. Effect of glucagon on esophageal motor function. Gastroenterology 1975;69:160-5.

7. Handal KA, Riordan W, Siese J. The lower esophagus and glucagon. Ann Emerg Med 1980;9:577-9.

8. Wong J, Cheung H, Lui R, Fan YW, Smith A, Siu KF. Esophagogastric anastomosis performed with a stapler: the occurrence of leakage and stricture. Surgery 1987;101:408-15.

9. Zilling TL, Walther BS, Johnsson F, von Holstein CS, Oberg S. Anastomotic diameter of circular stapled oesophagojejunal anastomoses and its implication for weight development. A clinical and experimental study. Eur J Surg 1995;161:193-8. 